\title{
Ressaut hydraulique ondulé : mythes et réalités
}

\section{H. Chanson}

Maitre de conférences en Hydraulique et Mécaniques des Fluides, Department of Civil Engineering, The University of Queensland, Brisbane QLD 4072, Australie

\section{IINTRODUCTION}

Pour un écoulement à surface libre, le passage d'un régime d'écoulement torrentiel (supercritique) à un régime d'écoulement fluvial (souscritique) s'appelle un ressaut hydraulique. Un ressaut est caractérisé par la présence de larges tourbillons et remous, des projections de paquets d'eau à la surface libre, un entraînement de bulles d'air et une dissipation importante d'énergie. Les conditions en aval d'un ressaut se déduisent des conditions d'écoulement amont, en combinant l'équation de conservation de la masse et l'équation de conservation de la quantité de mouvement. Pour un canal rectangulaire horizontal, de largeur constante, et en négligeant le frottement au fond et sur les parois du canal, on obtient :

$$
\begin{aligned}
& \frac{d_{2}}{d_{1}}=\frac{1}{2} *\left(\sqrt{1+8 * F r_{1}^{2}}-1\right) \\
& \frac{F r_{2}}{F r_{1}}=\frac{2^{3 / 2}}{\left(\sqrt{1+8 * F r_{1}^{2}}-1\right)^{3 / 2}}
\end{aligned}
$$

où les indices 1 et 2 indiquent, respectivement, les conditions d'écoulement en amont et en aval du ressaut (fig. l). La définition du nombre de Froude est : $F r=q_{w} N \sqrt{g * d^{3}}$.

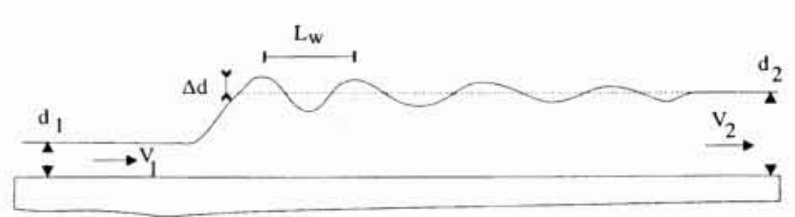

1. Déroulement d'un ressaut hydraulique ondulé.

L'équation de conservation d'énergie permet de calculer la dissipation d'énergie dans le ressaut :

$$
\frac{\Delta H}{d_{c}}=\frac{\left(d_{2}-d_{1}\right)^{3}}{4 * d_{1} * d_{2} * d_{c}}
$$

où $\Delta H$ est la perte de charge et $d_{c}$ est la profondeur critique. Pour un canal rectangulaire, la profondeur critique est égale à : $d_{c}=\sqrt[3]{\mathrm{qw}^{2} / g}$.

Si l'écoulement, en amont du ressaut, est critique $(F r=1)$ ou légèrement supercritique, le ressaut hydraulique ne déferle pas, mais il est suivi de longues ondulations de la surface libre; ces vagues sont stationnaires, à emplacement fixe. Un tel ressaut hydraulique est appelé un ressaut ondulé (« undular jump »). Pour un ressaut ondulé,

\section{Undular hydraulic jump : myths and reality}

In open channels, the transition from supercritical to subcritical flows is called a hydraulic jump. For low upstream Froude numbers, free-surface undulations develop downstream of the jump. Such a hydraulic jump is called an undular jump. New experiments on undular hydraulic jumps were performed in a rectangular channel in which the upstream flows were fully developed turbulent shear flows. The experimental data include free-surface profiles, velocity distributions, pressure distributions and local head profiles upstream and downstream of undular jumps. Visual and photographic observations indicate five types of undular jumps. For $\mathrm{Fr}>1.2$, the first wave crest is characterised by the development of lateral shock waves. The analysis of the data shows that the jump characteristics (e.g. wave length, wave amplitude) are functions of the upstream Froude number and the blockage ratio $d_{c} / W$. The disappearance of the undular jump is observed for Froude numbers ranging from 1.5 to 2.9 . 


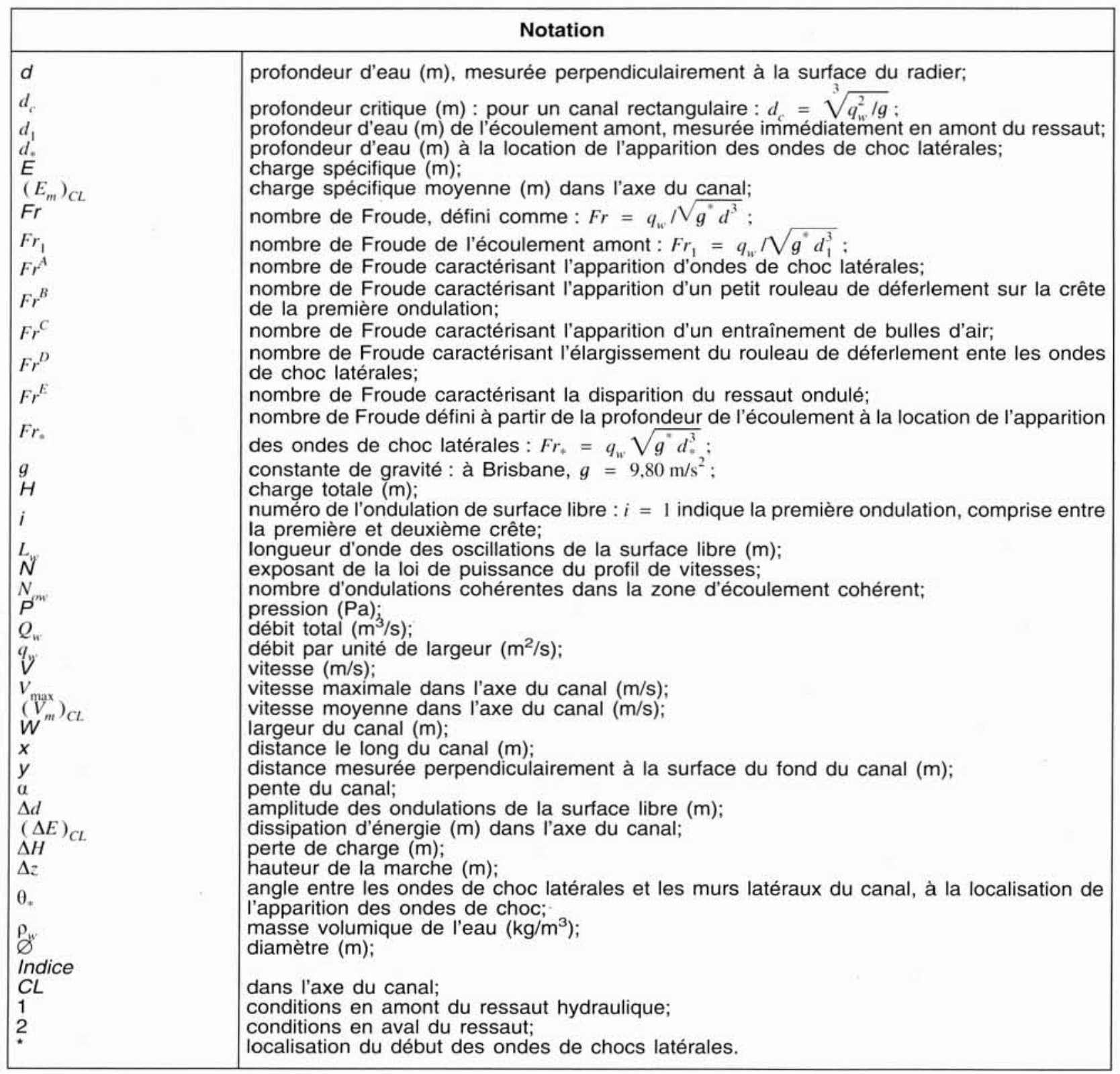

la perte de charge est très faible (eq. (3)) mais non nulle. En l'absence d'un rouleau de déferlement (« roller»), l'énergie dissipée est propagée, en aval, sous la forme de trains d'ondes stationnaires [1].

Dans cet article, on présente une revue des connaissances sur le ressaut ondulé (« Mythes »). Puis de nouveaux résultats expérimentaux sont présentés («Réalités»). Ces résultats sont ensuite discutés et comparés avec les résultats antérieurs («Mythes et Réalités »).

\section{II $\square$ MYTHES - REVUE BIBLIOGRAPHI- QUE}

La plupart des manuels d'hydraulique et livres universitaires, sur les écoulements à surface libre, ne traitent pas le cas des ressauts ondulés. Quelques ouvrages plus spé- cialisés [2], [3], [4] mentionnent l'existence du ressaut ondulé pour $F r_{1}<1,7$, mais n'en détaillent pas les propriétés.

Des études expérimentales, portant sur les ressauts hydrauliques, ont été faites avec quelques cas de ressauts ondulés : par exemple Bachmeteff et Matzke [5], Binnie et Orkney [6], Sandover et Holmes [7]. Mais ces chercheurs n'ont pas détaillé les propriétés spécifiques des ressauts ondulés. D'autres chercheurs ont étudié plus spécialement les ressauts ondulés : Lemoine [8], Serre [9], Iwasa [10], Jones [11], Andersen [12]. Cependant, toutes ces analyses sont basées sur une analogie avec la propagation d'ondes de translation dans un canal avec un fluide initialement au repos. De plus, les différents résultats obtenus sont parfois contradictoires.

Montes [1] a montré que l'analogie, entre le ressaut ondulé et les ondes de translation, ne tient pas compte des caractéristiques physiques de l'écoulement. Pour un res- 
saut ondulé, l'écoulement amont est caractérisé par un développement partiel, ou total, de la couche limite turbulente, sur le fond et les parois latérales ; ce qui n'est pas le cas pour les ondes positives de translation. De plus, les ondulations de la surface libre se propagent sur de longues distances, et l'hypothèse de frottements nuls (ou négligeables), utilisée dans la théorie des ondes de translation, n'est pas valide.

Récemment, Montes [13] et Ryabenko [14] ont présenté deux études pertinentes, menées indépendamment en Australie et en Union Soviétique. Leurs résultats contredisent le principe d'une analogie entre le ressaut ondulé et les ondes positives de translation. Ils suggèrent, même, que la transition entre un ressaut ondulé et un ressaut déferlant peut se produire pour des nombres de Froude compris entre 1,0 et 3,6 , en fonction des conditions d'écoulement amont.

\section{III — RÉALITÉS - MESURES EXPÉRIMEN- TALES}

\subsection{Description de l'expérimentation}

Plusieurs séries de mesures ont été menées dans un canal rectangulaire, long de 20 mètres. La largeur du canal est $0,25 \mathrm{~m}$, et la hauteur des parois latérales est $0,27 \mathrm{~m}$. Le fond et les parois latérales sont constitués de panneaux vitrés, long chacun de $1,25 \mathrm{~m}$. La pente du canal est ajustable, entre 0 et 4,5 degrés.

L'alimentation en eau du canal est assurée par un réservoir à niveau constant. Le débit est contrôlé par une vanne de réglage, installée sur le conduit d'alimentation reliant le réservoir à l'entrée du canal. A l'extrémité aval du canal, une vanne radiale permet de réguler l'écoulement à surface libre. La face amont de la vanne est recouverte d'une surface d'amortissement, pour empêcher la réflexion d'ondes et de vagues.

La mesure de débit est obtenue en mesurant la différence de pression au travers d'un coude à 90 degrés, dans la conduite d'alimentation. Pour les faibles débits $\left(Q_{W}<10 \ell / \mathrm{s}\right)$, le débit est déduit à partir du temps de remplissage d'un réservoir calibré de $300 \ell$. La position de la surface libre est mesurée avec une pointe, montée sur un chariot de translation. Les mesures de vitesses, pression et charge spécifique sont faites avec un tube de Pitot. Le diamètre extérieur du tube est : $\varnothing=3,3 \mathrm{~mm}$, et l'extrémité amont est percée d'un orifice de $1 \mathrm{~mm}$ de diamètre. Les prises de pression sont situés à $20 \mathrm{~mm}$ de l'extrémité, et elles ont un diamètre de $0,5 \mathrm{~mm}$. L'erreur sur la position verticale du tube de Pitot et de la pointe est inférieure à $0,2 \mathrm{~mm}$.

\subsection{Conditions d'écoulement amont}

Pour toutes ces mesures, on a positionné le ressaut ondulé à une distance minimale de $10 \mathrm{~m}$, depuis l'extrémité amont du canal. Cette disposition permet d'obtenir une couche limite turbulente complètement développée, où l'épaisseur de la couche limite égale la profondeur d'eau. Ce fait a été confirmé par toute une série de mesures de profils de vitesses, dans l'axe du canal, à 10 mètres en aval de l'entrée de canal.
La figure 2 montre plusieurs distributions de vitesses, dans l'axe du canal, à $10,10 \mathrm{~m}$ de l'extrémité amont du canal. La couche limite est complètement développée, et la distribution verticale des vitesses suit une loi polynomiale :

$$
\frac{V}{V_{\max }}=\left(\frac{y}{d}\right)^{1 / N}
$$

où $V_{\max }$ est la vitesse à la surface libre (dans l'axe du canal), $y$ est la distance depuis le fond de canal, mesurée perpendiculairement au fond, et $d$ est la profondeur d'eau.

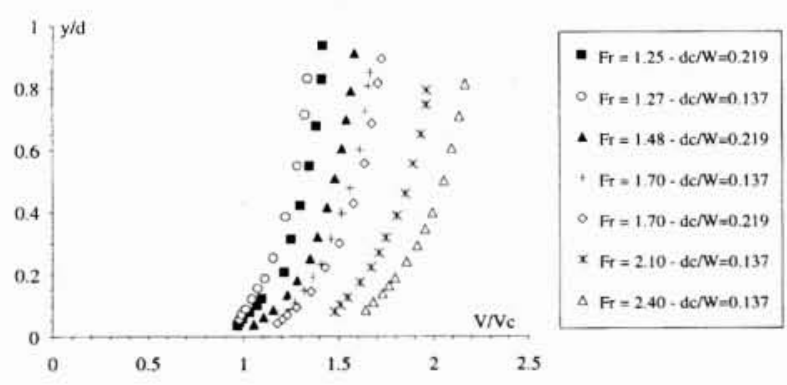

2. Distributions des vitesses en amont des ressauts ondulés.

Une analyse détaillée de ces mesures de vitesses [15] indique que le rapport de la vitesse moyenne $\left(V_{m}\right)_{C L}$, dans l'axe du canal, sur la vitesse moyenne $Q_{W} /\left(d^{*} W\right)$, est compris entre 1,05 et 1,35 : ce résultat suggère que les couches limites, le long des parois latérales du canal, induisent des effets tri-dimensionnels non-négligeables.

La même série de mesures indique, également, que la distribution de pression, à 10 mètres en aval de l'entrée du canal, est hydrostatique :

$$
P=\rho_{w} * g *(d-y) * \cos \alpha
$$

où $P$ est la pression, $\alpha$ est la pente du canal.

\subsection{Profils de la surface libre}

La figure 3 présente un profil typique de surface libre dans l'axe du canal. L'analyse des résultats expérimentaux montre que :

1) La longueur d'onde des ondulations de la surface libre est maximale à la première ondulation et décroit exponentiellement avec la distance. Cette décroissance est indépendante du nombre de Froude et du rapport $d_{c} / W$. La longueur d'onde de la $i$-ème ondulation $\left(L_{w}\right)$ peut être estimée en fonction de la longueur initiale $\left(L_{w_{1}}\right)$ par (Chanson [15]) :

$$
\frac{L_{w_{i}}}{L_{w_{1}}}=(i)^{-1 / 5}
$$

2) L'amplitude des ondulations est maximale à la première ondulation. La décroissance de l'amplitude des vagues est faible, et parfois nulle. 


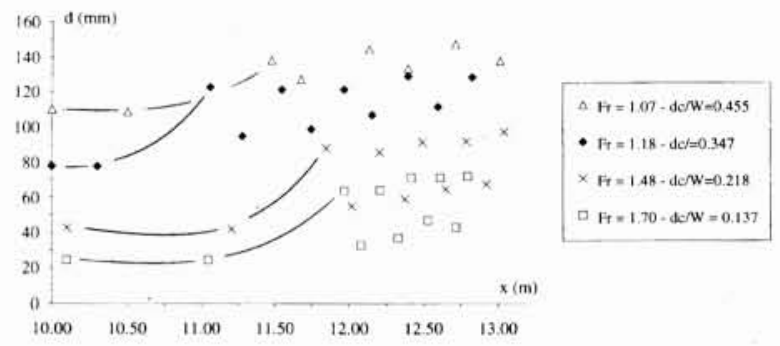

3. Exemples de profils de surface libre dans l'axe du canal.

3.4 Distributions de pressions, vitesses et énergies spécifiques

Différentes mesures de pressions, vitesses et charges spécifiques ont été faites, dans l'axe du canal, en amont du ressaut, aux crêtes des première, deuxième et troisième ondulations, aux ventres des première et deuxième ondulations, et à la naissance des ondes de choc latérales (dénotée onde de choc).

La figure 4 montre un résultat typique. La figure $4 a$ présente les distributions adimensionnelles de pression $\left(P /\left(\rho_{w} * g * d\right)_{C L}\right)$. On note que les distributions de pressions ne sont pas hydrostatiques. Le gradient moyen de pression $\mathrm{d} P / \mathrm{d} y$ est supérieur au gradient de pression hydrostatique $\left(\rho_{w} * g * \cos \alpha\right)$ aux ventres des vagues; il est inférieur au gradient hydrostatique à la hauteur des crêtes des vagues.

Les distributions de vitesses (fig. $4 b)$ sont présentées sous forme adimensionnelle, $V /\left(V_{m}\right)_{C L}$, où $\left(V_{m}\right)_{C L}$ égale :

$$
\left(V_{m}\right)_{C L}=\left(\frac{1}{d} * \int_{y=0}^{y=d} V * \mathrm{~d} y\right)_{C L}
$$

On remarque une modification très importante de la répartition des vitesses entre l'écoulement amont (loi polynomiale) et la première crête.

La figure $4 c$ montre les distributions de charges spécifiques $E /\left(E_{m}\right)_{C L}$, où $E$ et $\left(E_{m}\right)_{C L}$ sont définies respectivement comme :

$$
\begin{gathered}
E=\frac{P}{\rho_{w} * g}+y * \cos \alpha+\frac{V^{2}}{2 * g} \\
\left(E_{m}\right)_{C L}=\frac{1}{\left(V_{m}\right)_{C L}} *\left(\frac{1}{d} * \int_{y=0}^{y=d} E * V * \mathrm{~d} y\right)_{C L}
\end{gathered}
$$

\section{IV — MYTHES ET RÉALITÉS - DISCUSSION DES RÉSULTATS EXPÉRIMENTAUX}

\subsection{Classification du ressaut ondulé}

Pour des ressauts hydrauliques ondulés, avec une couche limite amont entièrement développée, les observations visuelles et photographiques, menées par l'auteur, mettent en évidence cinq (5) catégories de ressauts ondulés (fig. 5, tabl 1).
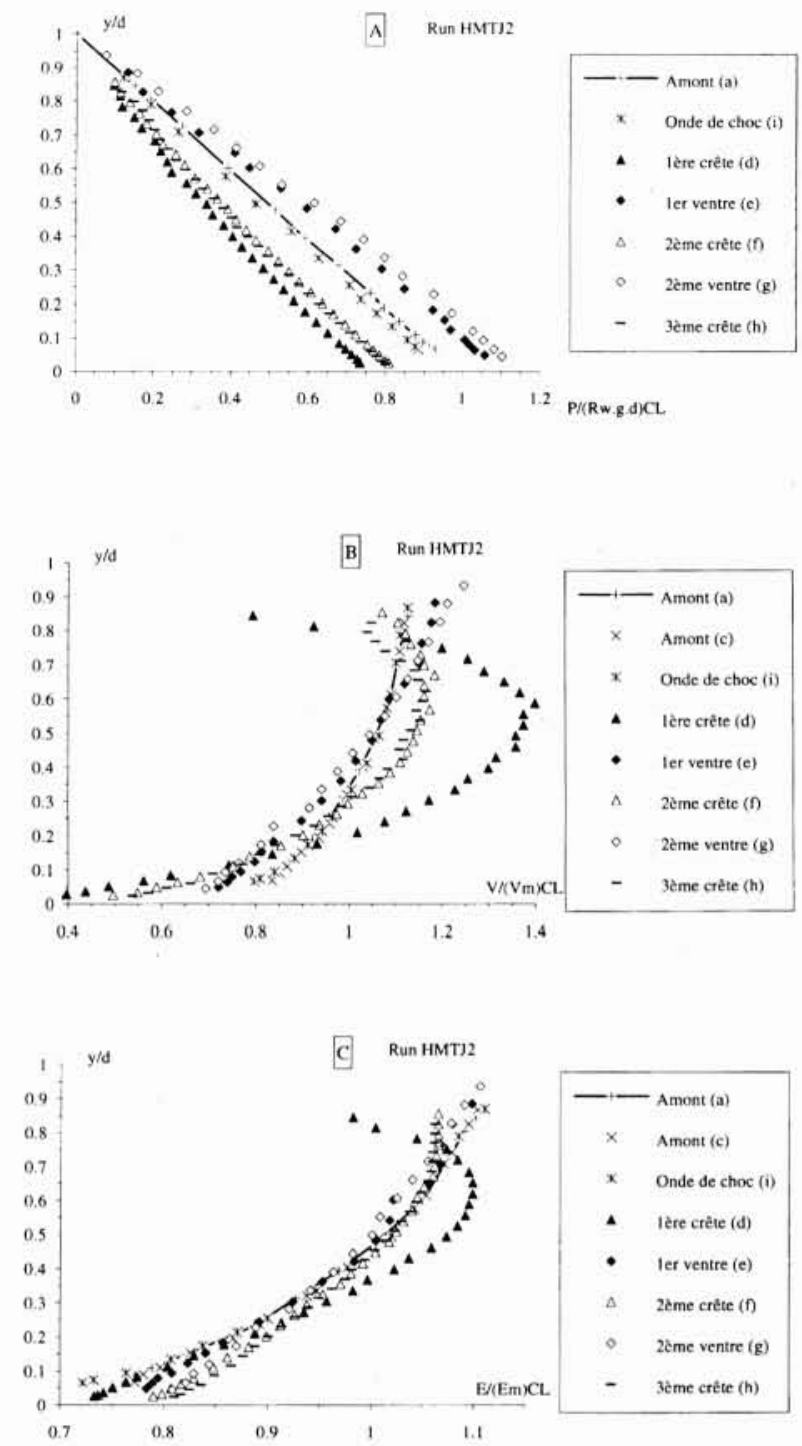

4. Distributions de pression, vitesses et charges spécifiques dans l'axe du canal au long d'un ressaut ondulé $q_{w}=0,02 \mathrm{~m}^{2} / \mathrm{s}, F r=1,70, d_{c} / W=0,138$, run HMTJ2

(A) distributions de pressions $P /\left(\rho_{w} * g * d\right)_{C L}$

(B) distributions de vitesses $V /\left(V_{m}\right)_{C L}$

(C) distributions de charges spécifiques $E /\left(E_{m}\right)_{C L}$.

A faibles nombres de Froude $\left(1 \leqslant F r_{1} \leqslant F r^{A}\right)$, l'écoulement est parfaitement bi-dimensionnel. Les ondulations de la surface libre sont de faibles amplitudes, avec de grandes longueurs d'ondes (fig. $6 \mathrm{a}$ ). Une augmentation du nombre de Froude $\left(F r^{A} \leqslant F r_{1} \leqslant F r^{B}\right)$ entraîne l'apparition d'ondes de choc latérales (ondes de Mach), se développant à la surface libre, depuis les parois latérales, en amont de la première crête (fig. $6 b$ ). Ces ondes intersectent très légèrement en aval de la première crête ; elles se réfléchissent sur le mur opposé, à la hauteur du creux 


\section{$\underline{\text { Type A }}$}

lère crête

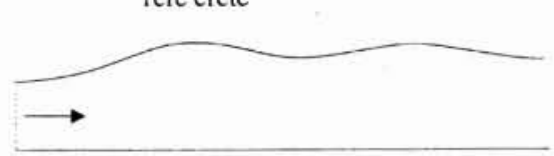

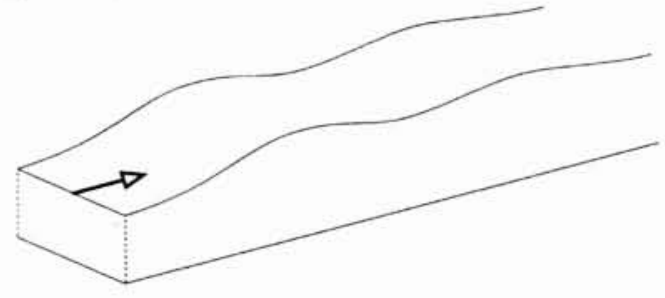

Type B

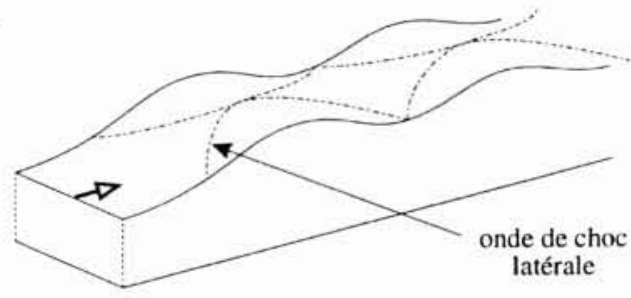

$\underline{\text { Type C }}$

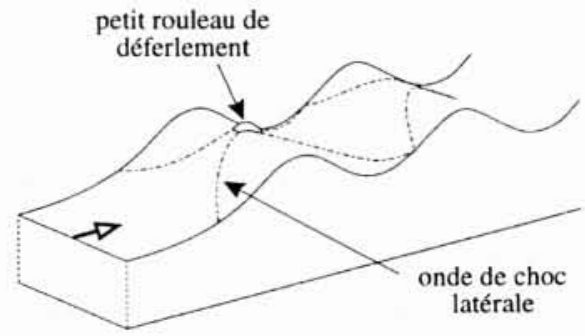

Type D

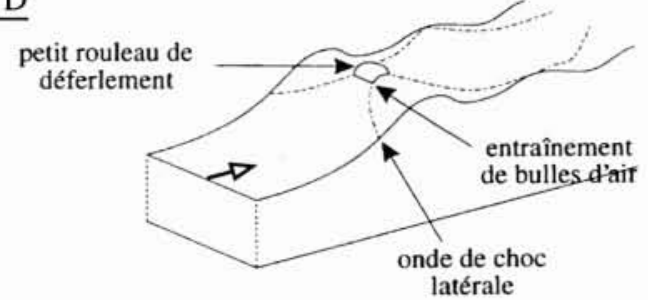

Type E

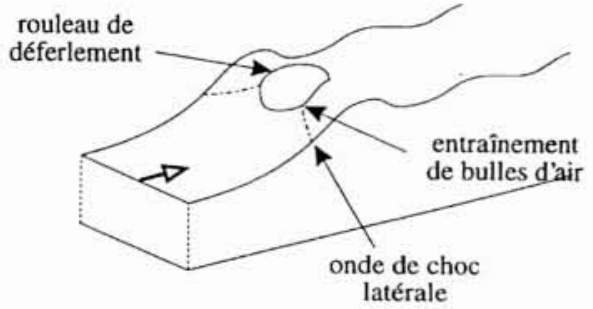

5. Différentes catégories de ressauts ondulés. 
Tableau 1. - Classification des ressauts ondulés.

\begin{tabular}{|c|c|c|c|c|c|}
\hline \multirow{2}{*}{$\begin{array}{c}\text { Caractéristique } \\
\text { (1) }\end{array}$} & \multicolumn{5}{|c|}{ Ressaut ondulé } \\
\hline & $\begin{array}{c}\text { Type A } \\
\text { (2) }\end{array}$ & $\begin{array}{l}\text { Type B } \\
\text { (3) }\end{array}$ & $\begin{array}{l}\text { Type C } \\
\text { (4) }\end{array}$ & $\begin{array}{l}\text { Type D } \\
\text { (4) }\end{array}$ & $\begin{array}{l}\text { Type } E \\
(6)\end{array}$ \\
\hline $\begin{array}{l}\text { Nombre de Froude } \\
F r_{1}:\end{array}$ & $1 \leqslant F r_{1} \leqslant F r^{A}$ & $F r^{A} \leqslant F r_{1} \leqslant F r^{B}$ & $F r^{B} \leqslant F r_{1} \leqslant F r^{C}$ & $F r^{C} \leqslant F r_{1} \leqslant F r^{D}$ & $F r^{D} \leqslant F r_{1} \leqslant F r^{E}$ \\
\hline Ecoulement : & $\begin{array}{c}\mathrm{Bi}- \\
\text { dimensionnel }\end{array}$ & $\begin{array}{c}\text { Tri- } \\
\text { dimensionnel }\end{array}$ & $\begin{array}{c}\text { Tri- } \\
\text { dimensionnel }\end{array}$ & $\begin{array}{c}\text { Tri- } \\
\text { dimensionnel }\end{array}$ & $\begin{array}{c}\text { Tri- } \\
\text { dimensionel }\end{array}$ \\
\hline $\begin{array}{l}\text { Ondes de choc } \\
\text { ("Mach") latérales: }\end{array}$ & Non & Oui & Oui & Oui & Oui \\
\hline $\begin{array}{l}\text { Rouleau de déferle- } \\
\text { ment: }\end{array}$ & Non & Non & $\begin{array}{l}\text { Oui } \\
\text { (petit) }\end{array}$ & $\begin{array}{c}\text { Oui } \\
\text { (moyen) }\end{array}$ & $\begin{array}{c}\text { Oui } \\
\text { (important) }\end{array}$ \\
\hline Entraînement d'air : & Non & Non & Non & $\begin{array}{c}\text { Oui } \\
\text { (faible) }\end{array}$ & $\begin{array}{c}\text { Oui } \\
\text { (moyen) }\end{array}$ \\
\hline $\begin{array}{l}\text { Région d'écoulement } \\
\text { permanent (cohé- } \\
\text { rent): }\end{array}$ & Très longue & Longue & Moyenne & Courte & Très courte \\
\hline $\begin{array}{l}\text { Région d'écoulement } \\
\text { unstationnaire (chao- } \\
\text { tique): }\end{array}$ & $(--)$ & $(--)$ & Courte & Longue & Longue \\
\hline
\end{tabular}

(--) : ne peut pas être observée dans le canal de mesure.

de la première vague, et intersectent de nouveau sur la deuxième crête. Vue d'en-haut, l'écoulement, en aval de la première crête, apparaît comme une succession de losanges, alignés dans l'axe du canal.

Pour $F r^{B} \leqslant F r_{1} \leqslant F r^{C}$, un petit rouleau de déferlement apparaît à l'intersection des ondes de Mach latérales, sur la première crête (fig. $6 c$ ). Ce petit rouleau a une forme de "crête de $\operatorname{coq}$ ". Pour des nombres de Froude supérieurs $\left(F r^{C} \leqslant F r_{1} \leqslant F r^{D}\right)$, le rouleau devient plus important, et des bulles d'air sont entraînées à l'intersection des ondes de choc et du petit rouleau, sur la première crête. Juste avant la disparition du ressaut ondulé $\left(F r^{D} \leqslant F r_{1} \leqslant F r^{E}\right)$, le rouleau de déferlement s'élargit, et bloque le développement des ondes de choc latérales (fig. $6 d$ ). Une vue en élévation de la première crête montre une forme en $W$, avec le rouleau de déferlement dans l'axe du canal (fig. 5).

Il est à noter, que, pour les ressauts ondulés de types $C$, $D$ et $E\left(F r>F^{B}\right.$, tableau 1$)$, les observations visuelles indiquent que : 1) les ondes de choc latérales intersectent sur la crête de la première ondulation, mais 2) leurs intersections successives (en aval de la première crête) ne s'effectuent plus sur les crêtes avales. En effet, les longueurs d'onde des ondulations de la surface libre deviennent plus courtes que la distance, nécessaire aux ondes de choc, pour traverser le canal.

\subsubsection{Nombres de Froude caractéristiques}

La figure 7 et le tableau 2 résument les observations sur les transitions entre les différents types de ressauts ondulés. On a indiqué aussi des résultats obtenus par d'autres chercheurs.

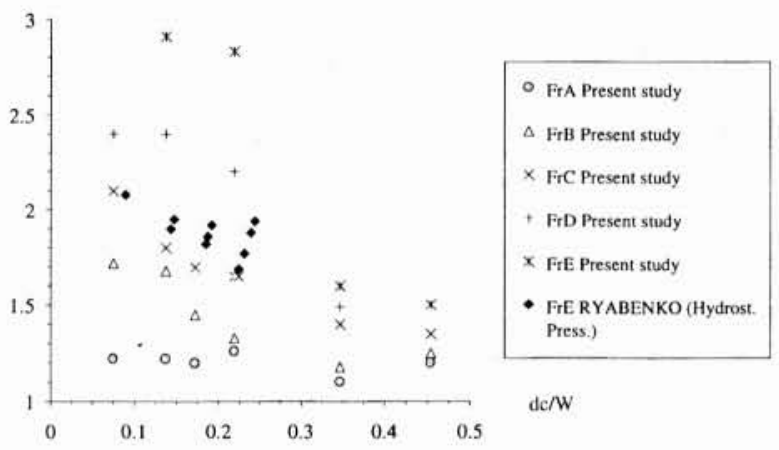

7. Nombres de Froude caractéristiques.

Il est intéressant de noter les travaux de Ryabenko [14], dans un canal de 1 mètre de largeur (tabl. 2). Ryabenko a utilisé différentes conditions d'écoulement amont : ses résultats suggèrent que la distribution de pression, en amont, a une influence importante sur les caractéristiques du ressaut ondulé.

\subsubsection{Disparition du ressaut ondulé}

Pour cette étude expérimentale, l'auteur définit la disparition du ressaut ondulé comme la disparition de la seconde longueur d'onde : c'est-à-dire quand il n'y a plus d'énergie propagée, en aval du ressaut, sous forme d'ondes stationnaires.

Les différents résultats expérimentaux (tabl. 2) suggèrent que le ressaut ondulé peut disparaître pour des nombres de Froude compris entre 1,0 et 3,6! Dans le cadre de cette nouvelle campagne de mesures, l'auteur a noté la disparition des ondulations, pour des nombres de Froude 

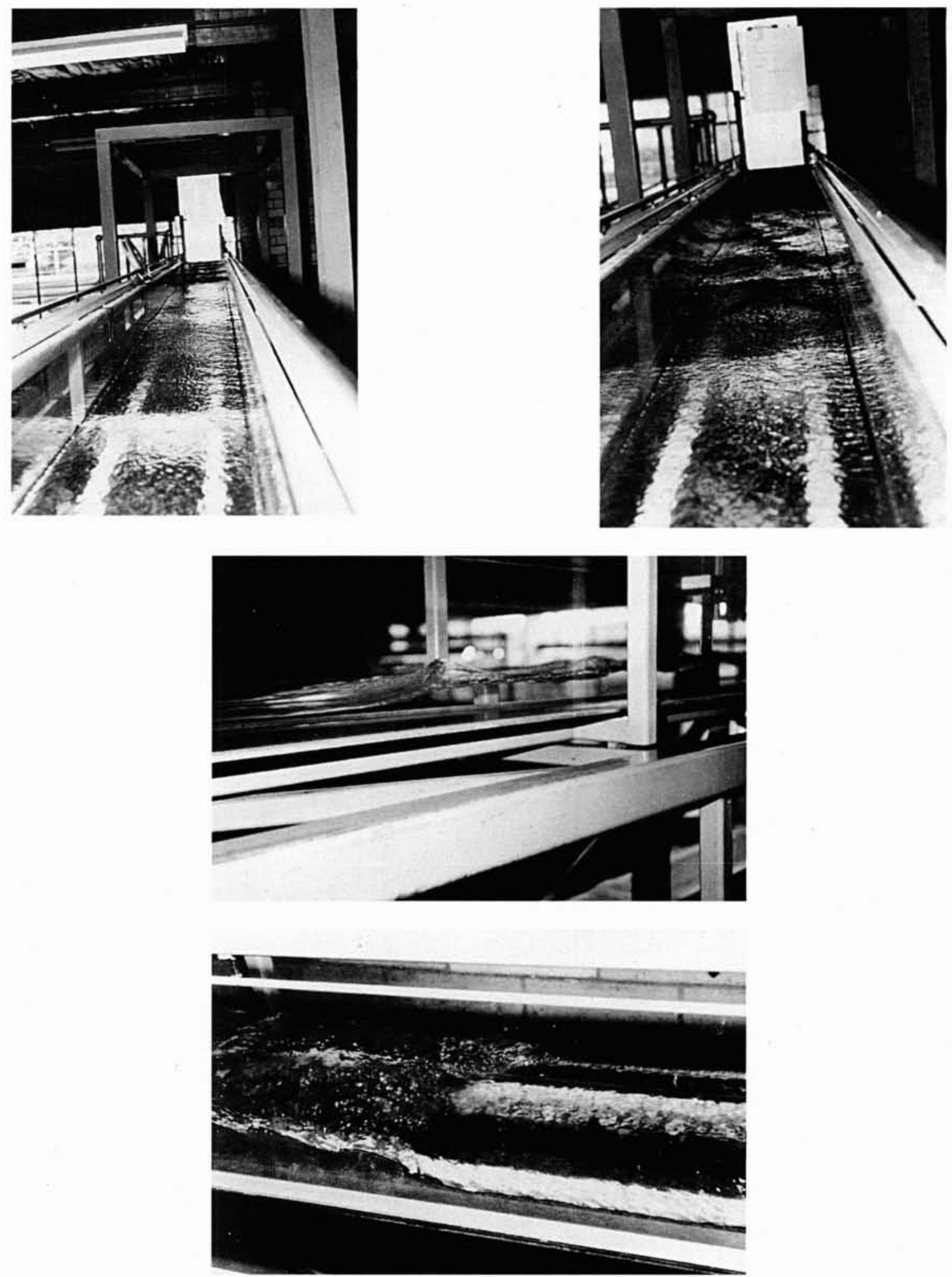

6. Photographies de ressauts ondulés

(a) ressaut ondulé type $\mathrm{A}-q_{w}=0,113 \mathrm{~m}^{2} \mathrm{~s}, F r_{1}=1,12, d_{c} / W=0,454$. Ecoulement de bas en haut; on note la première et la deuxième crête des vagues ;

(b) ressaut ondulé type $\mathrm{B}-q_{w}=0,08 \mathrm{~m}^{2} / \mathrm{s}, F r_{1}=1,36, d_{c} / W=0,347$. Ecoulement de bas en haut; on note les ondes de choc latérales, apparaissant en amont de la première crête ;

(c) ressaut ondulé type $\mathrm{C}-q_{w}=0,02 \mathrm{~m}^{2} / \mathrm{s}, F r_{1}=1,70, d_{c} / W=0,137$. Ecoulement de gauche à droite ; on remarque le petit rouleau de déferlement à l'intersection des ondes de choc ;

(d) ressaut ondulé type $\mathrm{E}-q_{w}=0,02 \mathrm{~m}^{2} / \mathrm{s}, F r_{1}=2,60, d_{c} / W=0,138$. Ecoulement de droite à gauche ; on note la taille importante du rouleau central, et on devine le début des ondes de choc latérales légèrement en amont de la première crête. 
Tableau 2. - Nombres de Froude caractéristiques.

\begin{tabular}{|c|c|c|c|c|c|c|c|}
\hline $\begin{array}{l}\text { Reference } \\
\text { (1) }\end{array}$ & $\begin{array}{l}d_{C} / W \\
(2)\end{array}$ & $\begin{array}{l}F r^{A} \\
\text { (3) }\end{array}$ & $\begin{array}{l}\mathrm{Fr}^{B} \\
(4)\end{array}$ & $\begin{array}{l}F r^{C} \\
(5)\end{array}$ & $\begin{array}{l}F r^{D} \\
(6)\end{array}$ & $\begin{array}{l}F_{r}^{E} \\
\text { (7) }\end{array}$ & $\begin{array}{l}\text { Remarques } \\
\text { (8) }\end{array}$ \\
\hline Etude présente & $\begin{array}{l}0,075 \\
0,137 \\
0,172 \\
0,219 \\
0,224 \\
0,347 \\
0,454\end{array}$ & $\begin{array}{l}1,22 \\
1,22 \\
1,2 \\
1,26 \\
1,10 \\
1,2\end{array}$ & $\begin{array}{l}1,72 \\
1,68 \\
1,45 \\
1,33 \\
1,18 \\
1,25\end{array}$ & $\begin{array}{l}2,10 \\
1,80 \\
1,7 \\
1,65 \\
1,65 \\
1,4 \\
1,35\end{array}$ & $\begin{array}{l}2,4 \\
2,4 \\
2,2 \\
1,49\end{array}$ & $\begin{array}{c}>2,57 \\
2,91 \\
2,83 \\
1,6 \\
1,5\end{array}$ & \\
\hline \multirow{2}{*}{$\begin{array}{l}\text { Ressauts ondulés } \\
\text { Ovalle et } \\
\text { Dominguez (1934) }\left({ }^{a}\right)\end{array}$} & & & & & & 1,59 & \\
\hline & & & & & & 3,63 & $\begin{array}{l}\text { Avec des } \\
\text { précautions } \\
\text { spéciales }\end{array}$ \\
\hline $\begin{array}{l}\text { Bakhmeteff } \\
\text { et Matzke (1936) }\left({ }^{b}\right)\end{array}$ & & & & & & 2,1 & $W=0.1524 \mathrm{~m}$ \\
\hline Serre (1953) & & & & & & 1,455 & Calcul théorique \\
\hline Binnie et Orkney (1955) & & & & & & 1,74 & \\
\hline \multirow[t]{2}{*}{ Iwasa (1955) } & & 1,5 & & & & 1,9 & Expérimentation \\
\hline & & & & & & 1.553 & Calcul théorique \\
\hline \multirow[t]{2}{*}{ Ryabenko (1990) } & $\begin{array}{l}0,090 \\
0,143 \\
0,147 \\
0,185 \\
0,187 \\
0,192 \\
0,224 \\
0,231 \\
0,239\end{array}$ & & & & & $\begin{array}{l}2,08 \\
1,90 \\
1,95 \\
1,82 \\
1,86 \\
1,92 \\
1,69 \\
1,77 \\
1,88\end{array}$ & $\begin{array}{l}\text { Distribution de } \\
\text { pression } \\
\text { hydrostatique } \\
\text { en amont du } \\
\text { ressaut } \\
W=1 \mathrm{~m}\end{array}$ \\
\hline & $\begin{array}{l}0,060 \\
0,109 \\
0,121 \\
0,010 \\
0,041 \\
0,043 \\
0,044 \\
0,124\end{array}$ & & & & & $\begin{array}{l}1,01 \\
1,34 \\
1,02 \\
1,0 \\
1,03 \\
1,25 \\
1,42 \\
1,61\end{array}$ & $\begin{array}{l}\text { Distribution de } \\
\text { pression non- } \\
\text { hydrostatique } \\
\text { en amont du } \\
\text { ressaut } \\
W=1 \mathrm{~m}\end{array}$ \\
\hline $\begin{array}{l}\text { Ressauts ondulés à une } \\
\text { marche arrondie } \\
\text { Sharp (1974) }\end{array}$ & & & & & & $\begin{array}{l}2,2 \\
3,5\end{array}$ & $\begin{array}{l}\Delta z / d_{1}=2 \\
\Delta z / d_{1}=4 \\
W=0,2 \mathrm{~m}\end{array}$ \\
\hline Hager et Kawagoshi (1990) & $\begin{array}{c}0,03 \text { to } \\
0,135\end{array}$ & & & & & $2,44+0,28 * \frac{\Delta z}{d_{1}}$ & $W=0,5 \mathrm{~m}$ \\
\hline
\end{tabular}

\section{(a) : Cité par Montes [13].}

(b) : Bakhmeteff et Matzke [5] indiquaient que le ressaut ondulé disparaissait pour $F r_{1}=1,732$. Mais la ré-analyse de leurs données expérimentales montre que les ondulations de la surface disparaissent pour $\operatorname{Fr}_{1}=2,1$.

$\Delta z$ : hauteur de la marche $(\mathrm{m})$.

compris entre 1,5 et 2,9 . Les résultats de l'auteur et de Ryabenko [14] montrent que la transition d'un ressaut ondulé à un ressaut déferlant (weak jump) est une fonction du rapport $d_{c} / W: F^{E}$ diminue quand le débit augmente pour une largeur de canal constante (fig. 7).

\subsection{Caractéristiques spécifiques d'un ressaut ondulé}

En comparaison avec les ondes positives de translation, un ressaut ondulé est caractérisé par : 1) la présence d'ondes 
de chocs latérales, 2) un profil longitudinal caractéristique, et 3 ) éventuellement des courants de recirculation secondaires.

\subsubsection{Ondes de choc latérales}

Quand le nombre de Froude $F r_{1}$ est pratiquement égal à $\mathrm{Fr}^{A}$, de faibles ondes de choc commencent à apparaître à l'intersection des parois latérales et de la surface libre, en amont de la première crête, mais ces ondes disparaissent en se propageant vers le centre du canal. Pour $F r_{1}>F r^{A}$, les ondes de choc s'étendent sur toute la largeur du canal, et se réfléchissent sur la paroi opposée (fig. 5). Ces ondes de Mach transversales intersectent, pour la première fois, sur la crête de la première vague (fig. $6 b, 6 c$, $6 d)$. Le front de ces ondes de choc n'est pas ondulé (undular shock wave) mais consiste en un rouleau de déferlement marqué (roller shock wave).

L'auteur a observé l'apparition des ondes de choc transversales lorsque $\mathrm{Fr}^{A}=1,2$ (fig. 7), indépendamment du rapport $d_{c} / W$. On notera que Iwasa [10] a observé, aussi, des ondes de chocs transversales, pour $F r^{A} \geqslant 1,5$. Montes [13] a suggéré que l'existence de ces ondes de choc est liée à l'interaction entre le ressaut et les couches limites créées par les parois latérales: les couches limites latérales retarderaient le fluide près des murs, et forceraient une apparition "prématurée " des conditions critiques $(F r=1)$, en comparaison du lit principal du canal.

Pendant les expérimentations, on a mesuré la profondeur de l'écoulement, $d_{s}$, à la naissance des ondes de choc, et l'angle, $\theta_{*}$, entre les ondes de chocs et les parois latérales. Les résultats sont présentés en figure 8 . On remarque que les ondes de choc latérales apparaissent dans une zone d'écoulement supercritique :

$$
F r_{*}=F r_{1}-0,122
$$

où $F r_{*}=q_{w} / \sqrt{g^{*} d_{*}^{3}}$. On note, aussi, que l'angle des ondes de choc, avec les murs latéraux, augmente sensiblement avec le nombre de Froude $F r_{1}$, avec des angles $\theta$. compris entre 20 et 40 degrés :

$$
\theta_{*}=33,32 * F r_{1}^{0,232}
$$

où $\theta$. est en degrés. Ces observations (eq. (11)) ne correspondent pas aux résultats classiques de la théorie des ondes de choc, dans les écoulements à surface libre [16] pour un écoulement supercritique, l'angle entre les ondes de choc et les parois latérales décroit avec le nombre de Froude.

L'auteur est persuadé que les ondes de choc latérales résultent d'interactions entre les couches limites latérales, la modification du profil des vitesses et la remontée progressive de la surface libre $(\mathrm{d} P / \mathrm{d} x>0)$. Mais il ne comprend pas les mécanismes fondamentaux de ces ondes de choc.

\subsubsection{Profil longitudinal}

Pour les ressauts ondulés de types $A$ et $B$, la surface libre en aval du ressaut est constituée d'ondulations stationnaires bi-dimensionnelles ou tri-dimensionnelles.
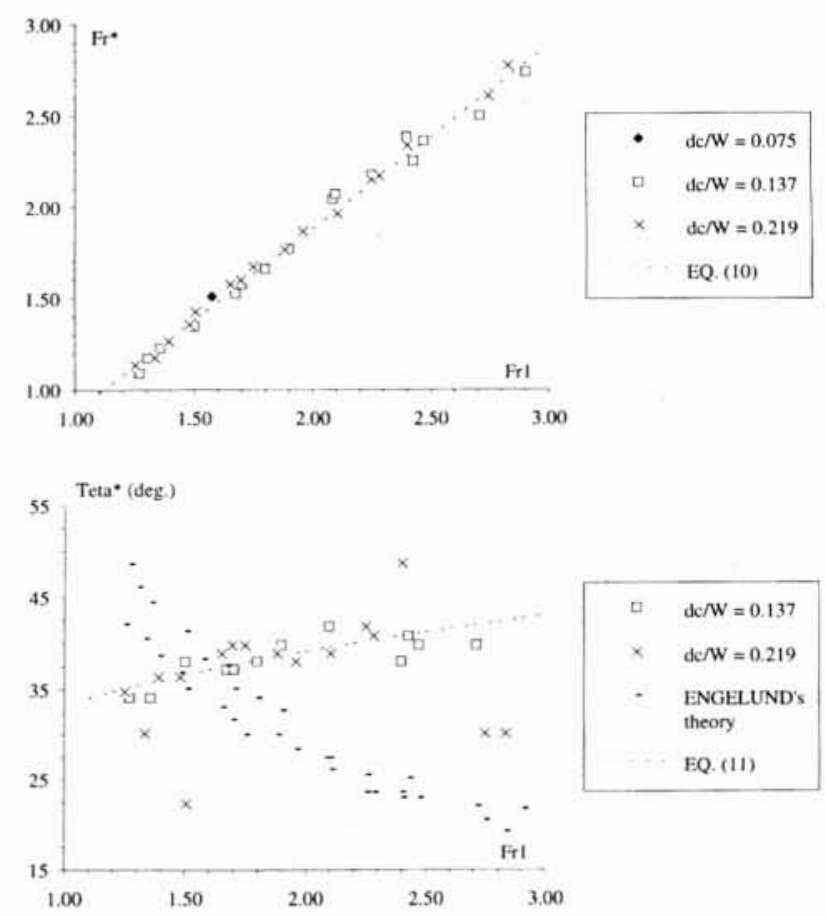

8. Caractéristiques du point d'inception des ondes de choc latérales ;

(a) $F r_{*}=q_{w} / \sqrt{g^{*} d_{*}^{3}}$ en fonction du nombre de Froude amont $\mathrm{Fr}_{1}$; (b) $\theta$, en fonction du nombre de Froude amont $F r_{1}$.

Par contre, pour de plus grands nombres de Froude $\left(F r_{1}>F r^{B}\right)$, la surface libre en aval du ressaut comprend, d'amont en aval (fig. 9): 1) une région d'écoulement permanent cohérent, 2) une région d'écoulement instationnaire et chaotique, et 3 ) une zone d'amortissement.

Dans la zone d'écoulement cohérent, chaque crête et chaque ventre des ondulations sont clairement marqués. Les amplitudes sont maximales dans l'axe du canal. La surface libre près des murs est caractérisée par des ondulations de même longueur d'onde et même phase que dans l'axe du canal, mais avec des amplitudes beaucoup plus faibles. Dans la région d'écoulement chaotique, les ondulations de la surface libre deviennent instationnaires, chaotiques et tri-dimensionnelles. Les amplitudes des oscillations de la surface libre sont maximales près des parois latérales, et elles sont du même ordre de grandeur que l'amplitude des premières ondulations en aval du ressaut (observées dans l'axe du canal).

Les observations montrent que la longueur de la zone d'écoulement cohérent décroit lorsque le nombre de Froude augmente. Pour $F r_{1}>F^{B}$, le nombre de longueurs d'ondes stationnaires et cohérentes peut être corrélé par :

$$
N_{o w}=\left(15,5+3,7 * \frac{d_{c}}{W}\right) *\left(F r^{E}-F r_{1}\right)
$$

Il est intéressant de noter, que Darcy et Bazin [17] et Hager et Hutter [18] ont observé aussi le comportement tri-dimensionnel de la surface libre. En particulier, la figure 20 de Darcy et Bazin [17] montre trois à cinq 


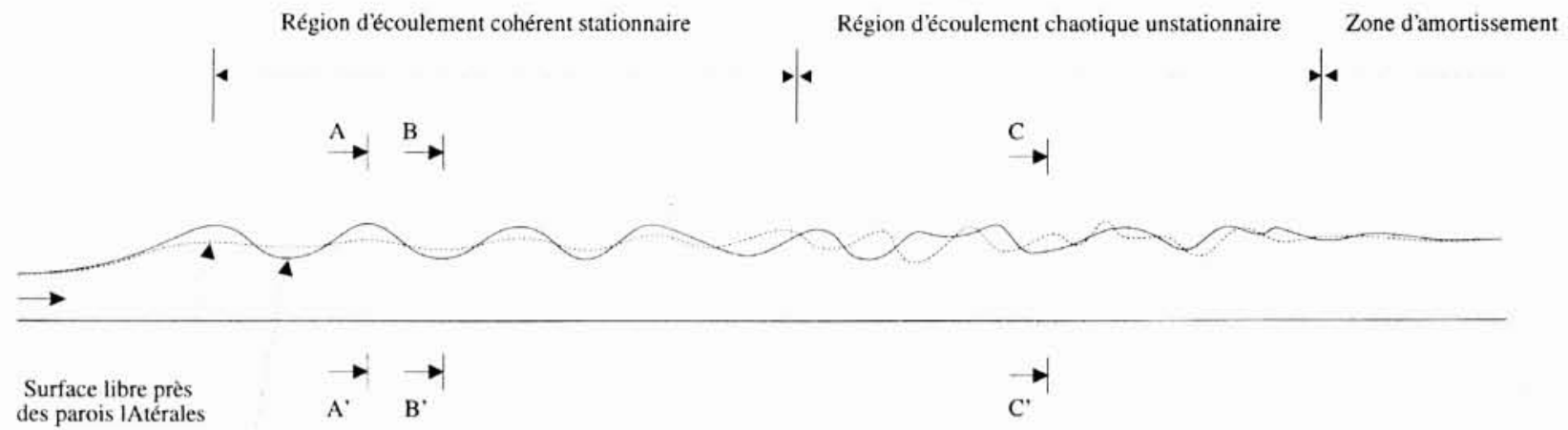

Surface libre dans l'axe du canal

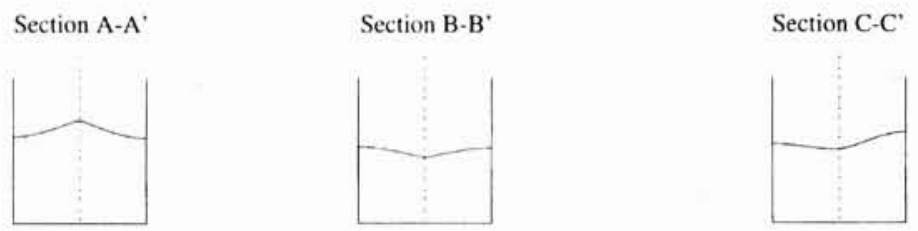

\section{Profil longitudinal d'un ressaut ondulé.}

ondulations stationnaires, suivies d'une amplification des ondulations près des parois latérales.

Lors de la conception d'un canal, l'instabilité de la surface libre près des parois latérales est le souci principal, déterminant la hauteur de ces parois. Des études complémentaires sont nécessaires pour quantifier plus précisément ces instabilités.

\subsubsection{Courants de recirculation}

Montes [13] a observé un décollement et une séparation près du fond du canal, à la hauteur de la première crête. L'auteur n'a pas observé ce type de recirculation pendant ses mesures. Il estime que ce courant de recirculation secondaire de fond peut exister pour des couches limites partiellement développées, mais n'existe pas avec une couche limite complètement développée.

Par contre, lorsque le nombre de Froude est suffisamment important, on a observé deux types de recirculation : une recirculation de surface, entre les ondes de choc et la crête de la première vague, et des courants secondaires dans les coins, à la hauteur de la première et deuxième crête (fig. 10).

Le premier type de recirculation est observé immédiatement en aval des ondes de choc latérales: le contrecourant de recirculation, d'axe de rotation vertical, est limité à une faible épaisseur d'eau, près de la surface libre. Il apparaît en même temps que les ondes de choc.

Les courants secondaires, dans les coins, à la hauteur des première et deuxième crêtes, sont indépendant de la recirculation de surface. L'auteur pense qu'ils sont induits par les interactions entre les couches limites du fond et des murs latéraux.

\subsection{Amplitudes des ondulations de la surface libre}

A partir des mesures des profils de la surface libre, il est possible de déduire l'amplitude des ondulations de la sur-
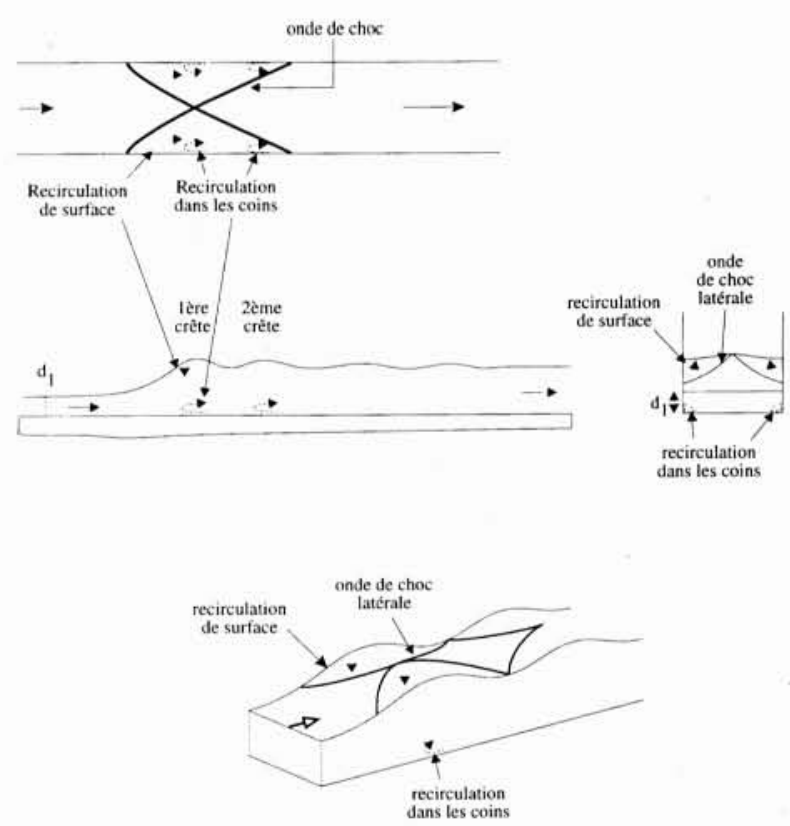

10. Courants de recirculation.

face libre. Les résultats expérimentaux sont présentés en figure 11. Et ils sont comparés avec les calculs théoriques pour les ondes de translation, initialement présentés par Boussinesq [14], et redéveloppés par Andersen [12].

Pour des écoulements faiblement supercritiques, les résultats obtenus avec des ressauts ondulés sont similaires à ceux obtenus avec les ondes de translation. Pour des nombres de Froude plus importants, l'amplitude des oscillations de la surface libre diverge des calculs d'ondes de translation, puis atteint une valeur maximale, puis décroit vers une valeur «plateau». 


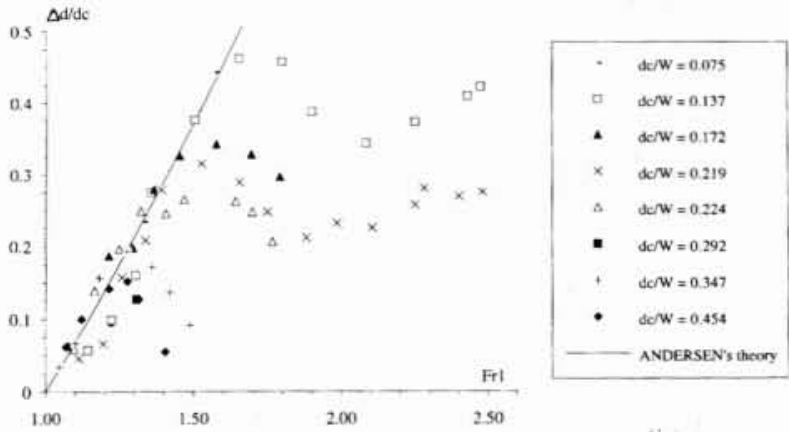

11. Amplitude $\Delta d / d_{c}$ de la première ondulation de la surface libre en fonction du nombre de Froude $F r_{1}$.

Les mesures expérimentales montrent que la relation entre l'amplitude des ondulations et le nombre de Froude n'est pas unique, mais dépend du rapport $d_{c} / W$. On note que l'amplitude des ondulations peut atteindre presque la moitié de la profondeur critique.

\subsection{Dissipation d'énergie}

L'analyse de l'ensemble des données expérimentales (reportées par Chanson [15]) montre, de manière consistente, que la plus grande partie de la dissipation d'énergie, dans l'axe du canal, a lieu immédiatement en amont de la première crête de vague : plus particulièrement, entre le point d'apparition des ondes de choc et la première crête. Au point d'apparition des ondes de choc latérales, le profil de pression (dans l'axe) est pratiquement hydrostatique (eq. (5)), et la distribution des vitesses est à peu près polynomiale (eq. (4)). Par contre, à la première crête, les profils de vitesses et pressions sont complètement perturbés et redistribués (fig. 4).

La figure 12 montre la dissipation d'énergie $(\Delta E)_{C L}$, observée dans l'axe du canal, entre l'écoulement amont et la première crête. $(\Delta E)_{C L}$ est égale à la perte de charge totale moins la différence d'élévation du fond du canal. Les résultats sont comparés avec l'équation (3).

Cette analyse suggère que la dissipation d'énergie, dans l'axe du canal, s'effectue, principalement, entre l'écoulement amont et la première crête des vagues. Toutefois, cette conclusion ne doit pas être étendue à l'écoulement moyenné du ressaut. En effet, ces séries de mesures ont montré le caractère tri-dimensionnel d'un ressaut ondulé, et de nouvelles mesures, près des parois latérales, sont nécessaires.

\section{VD CONCLUSION}

La transition entre un écoulement faiblement supercritique et un écoulement fluvial est caractérisé par le développement d'ondulations de la surface libre en aval du ressaut hydraulique : un tel ressaut s'appelle un ressaut ondulé.

L'auteur a présenté de nouveaux résultats expérimentaux de ressauts ondulés. Ces résultats ont été obtenus dans un canal rectangulaire, et les écoulements amonts étaient

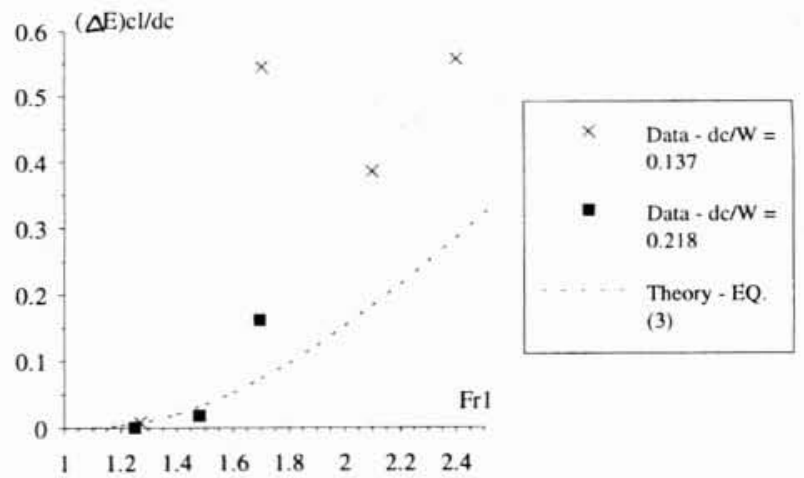

12. Dissipation d'énergie dans l'axe du canal d'écoulement amont et la première crête en terme adimensionnel $(\Delta E)_{C L} / d_{c}$. Comparaison avec l'équation (3).

turbulents, avec une couche limite entièrement développée. On a présenté des profils typiques de la surface libre, des mesures de pressions, vitesses et charges spécifiques au long de ressaut ondulés.

Les observations montrent que l'on peut définir cinq types de ressauts ondulés : un ressaut bi-dimensionnel pour $\mathrm{Fr}<1,2$, et, pour des nombres de Froude plus important, des ressauts tri-dimensionnels, caractérisés par des ondes de chocs latérales, un petit rouleau de déferlement ou un entraînement de bulles d'air (tabl. l). Une des caractéristiques spécifiques du ressaut ondulé est la présence d'ondes de choc latérales.

L'analyse des données expérimentales indique que l'amplitude des ondulations et leur disparition dépendent du nombre de Froude et du rapport de la profondeur critique sur la largeur de canal $d_{c} / W$. Pour cette étude, la disparition du ressaut ondulé a lieu pour des nombres de Froude compris entre 1,5 et 2,9 . On note, de plus, que, dans l'axe du canal, la majeure partie de l'énergie est dissipée entre le point d'apparition des ondes de choc latérales et la première crête.

\section{Remerciements}

L'auteur tient à remercier Professeur J. Fenton, Monash University (Australie), et Dr J.S. Montes, University of Tasmania (Australie), pour leurs encouragements et de fructueuses discussions.

Il remercie également Monsieur $\mathrm{H}$. Maroulier, qui a effectué plusieurs expérimentations, ainsi que I'ENSHM Grenoble (France), et le Conseil Général de l'Isère (France), pour le support financier de Monsieur Maroulier, lors de son stage.

\section{Bibliographie}

[1] Montes J.S. (1979). - Undular Hydraulic Jump - Discussion. Jl of Hyd. Div., ASCE, Vol. 105, No. HY9, pp. 1208-1211.

[2] Rouse H. (1938). - Fluid Mechanics for Hydraulic Engineers. McGraw-Hill, New York, USA.

[3] Chow V.T. (1959). - Open Channel Hydraulics. McGrawHill International, New York, USA. 
14] Henderson F.M. (1966). - Open Channel Flow. MacMillan Company, New York, USA.

[5] BAKhmetefF B.A. et MAtZKe A.E. (1936), - The Hydraulic Jump in Terms of Dynamic Similarity. Transactions, ASCE, Vol. 101, pp. 648-680.

[6] BINNIE A.M. et ORKNEY J.C. (1955). - Experiments on the Flow of Water from a Reservoir through an Open Channel. II. The Formation of Hydraulic Jump. Proc. of the Royal Soc., London, Series A, Vol. 230, pp. 237-245.

[7] SANdover J.A. et Holmes P. (1962). - The Hydraulic Jump in Trapezoidal Channels. Water Power, Nov., pp. 445449.

[8] Lemoine R. (1948). - Sur les Ondes Positives de Translation dans les Canaux et sur le Ressaut Ondulé de Faible Amplitude. (On the Positive Surges in Channels and on the Undular Jumps of Low Wave Height). Jl La Houille Blanche, Mar-Apr., pp. 183-185.

[9] SERRE F. (1953). - Contribution à l'Etude des Ecoulements Permanents et Variables dans les Canaux. (Contribution to the Study of Permanent and Non-Permanent Flows in Channels). JI La Houille Blanche, Dec., pp. 830-872.

[10] IwASA Y. (1955). - Undular Jump and its Limiting Conditions for Existence. Proc. of the 5th Japan National Congress for Applied Mechanics, Paper II-14, pp. 315-319.

[11] JONES L.E. (1964). - Some Observations on the Undular Jump. Jl of Hyd. Div., ASCE, Vol. 90, No. HY3, pp. 69-82.

[12] ANDERSEN V.M. (1978), - Undular Hydraulic Jump. Jl of Hyd. Div., ASCE, Vol. 104, No. HY8, pp. 1185-1188. Discussion: Vol. 105, No. HY9, pp. 1208-1211.

[13] MONTES J.S. (1986). - A Study of the Undular Jump Profile. Proc. of the 9th Australasian Fluid Mechanics Conference AFMC, Auckland, New Zealand, pp. 148-151.
[14] RyABEnko A.A. (1990). - Conditions Favorable to the Existence of an Undulating Jump. Gidrotekhnicheskoe Stroitel'stvo, No. 12, pp. 29-34 (en Russe). (Traduction anglaise: Hydrotechnical Construction, 1990, Plenum Publ., pp. 762-770).

[15] Chanson H. (1993). - Characteristics of Undular Hydraulic Jumps. Research Report No. CE, Dept. of Civil Engineering, University of Queensland, Australia.

[16] Engelund F. et Munch-Petersen J. (1953). - Steady Flow in Contracted and Expanded Rectangular Channels. Some Considerations Concerning the Shape of the Water Surface. JI La Houille Blanche, Aug./Sept., pp. 464-474.

[17] DARCY H. et BAZIN H. (1865). Recherches Hydrauliques. (Hydraulic Research). Imprimerie Impériale, Parties le et 2e, Paris, France.

[18] Hager W.H. et Hutter K. (1984). - On Pseudo-Uniform Flow in Open Channel Hydraulics. Acta Mechanica, Vol. 53. pp. 183-200.

[19] BoussinesQ J.V. (1871). - Sur le Mouvement Permanent Varié de l'Eau dans les Tuyaux de Conduite et dans les Canaux Découverts. (On the Steady Varied Flow of Water in Conduits and Open Channels). Comptes Rendus des séances de l'Académie des Sciences, Paris, France, Vol. 73. Jne 19, pp. 101-105.

[20] Hager W.H. et Kawagoshi N. (1990). - Hydraulic Jumps at Rounded Drop. Proc. Instn. Civ. Engrs, Part 2, Vol. 89. Dec., pp. 443-470.

[21] Ovalle A. et Dominguez A. (1934). - Civil Engineering Thesis. Catholic University, Santiago, Chile.

[22] Sharp J.J. (1974). - Observations on Hydraulic Jumps at Rounded Steps. Jl of Hyd. Div., ASCE, Vol. 100, No. HY6, pp. 787-795. 